Check for updates

Cite this: RSC Adv., 2018, 8, 38787

Received 9th August 2018

Accepted 2nd November 2018

DOI: $10.1039 / \mathrm{c} 8 \mathrm{ra06693k}$

rsc.li/rsc-advances

\section{A lipopeptide biosurfactant from Bacillus sp. Lv13 and their combined effects on biodesulfurization of dibenzothiophene $\uparrow$}

\author{
Yinghai Lyu, (D) *a Tingting Zhang, ${ }^{\mathrm{b}}$ Baojuan Dou, ${ }^{\mathrm{C}}$ Guijiang Li, ${ }^{\mathrm{a}}$ Chengxin Ma \\ and Yangyang $\mathrm{Li}^{\mathrm{a}}$
}

\begin{abstract}
The process of using biodesulfurization (BDS) to remove sulfur compounds in petroleum has limitations such as low efficiency and low mass transfer. Therefore, it is important to study the combined effects of biosurfactant and the strain on BDS. A thermophilic desulfurization strain, Bacillus sp. Lv13, was isolated from the oilfield and used to produce biosurfactant (BS). The strain was identified as Bacillus licheniformis, a moderate thermophilic bacterium. Its BS was identified as lipopeptide using thin-layer chromatography (TLC), gas chromatography-mass spectrometry (GC-MS) and Fourier transform infrared spectroscopy (FT-IR). The emulsification efficiency after $24 \mathrm{~h}\left(E_{24}\right)$ and critical micelle concentration (CMC) were determined to be $46.93 \%$ and $30 \mathrm{mg} \mathrm{L}^{-1}$, respectively. The combined effects of biosurfactant and the strain on BDS was confirmed using the Gibbs assay, GC-MS and $\mathrm{BaCl}_{2}$ test. Results showed that the yield of 2-hydroxybiphenyl (2-HBP) from dibenzothiophene significantly increased after the addition of lipopeptide into the reaction system. This could be illustrated by the stabilization of emulsion, lower CMC value, higher mass transfer rate with the addition of lipopeptide, and the enhancement in the capacity of BDS as well as the catalytic ability of the microbial cell.
\end{abstract}

\section{Introduction}

As a modern industrial "blood", petroleum has been widely used in the world. However, the sulfur compounds in petroleum causes equipment corrosion, catalyst poisoning, and environmental problems. Therefore, it is very important to research the performance of desulfurization of petroleum. Desulfurization methods are mainly categorized into hydrodesulfurization (HDS) and non-hydrodesulfurization. HDS requires higher temperatures and pressures $^{1}$ while biodesulfurization (BDS), one important non-hydrodesulfurization method, can be carried out under ambient temperature and pressure. ${ }^{2}$ BDS has the advantages of being environmental friendly, requiring less energy intensity, and having a low cost. Dibenzothiophene (DBT) is often used as the model compound for BDS. Some strains can selectively convert DBT to 2-hydroxybiphenyl (2HBP). ${ }^{3}$ This metabolic pathway was named the " $4 \mathrm{~S}$ " pathway, which has the advantage of maintaining the calorific value of

${ }^{a}$ Department of Bioengineering, College of Chemical and Environmental Engineering, Shandong University of Science and Technology, Qingdao 266590, PR China. E-mail: yhlv666@163.com; Fax: +86-0532-80681197; Tel: +86-0532-86057106

${ }^{b}$ College of Chemical and Environmental Engineering, Shandong University of Science and Technology, Qingdao 266590, PR China

${ }^{c}$ College of Biotechnology and Bioengineering, Zhejiang University of Technology, Hangzhou, 310014, PR China

$\dagger$ Electronic supplementary information (ESI) available. See DOI: $10.1039 / \mathrm{c} 8 \mathrm{ra06693k}$ the petroleum. Biosurfactant (BS), a surface-active metabolite secreted by microorganisms, exhibits excellent characteristics such as oil recovery and bioremediation due to its amphiphilic structure. ${ }^{4}$ For example, the combination of biosurfactantproducing strains and petroleum-degrading strains exhibited a higher petroleum biodegradation efficiency of $85.4 \%$ compared to that of only petroleum-degrading strains $(71.2 \%){ }^{5}$ The solubility of water-immiscible sulfur nutrients increased due to the formation of micelles and eased the uptake due to bacteria localization in the emulsion layer. ${ }^{6-8}$ The addition of surfactants to the catalytic system formed by non-immobilized or immobilized cells increased the desulfurization of DBT and gas oil. ${ }^{9}$ However, there are relatively few detailed reports focusing on the BS effect of the BDS of DBT. The improvement of bacterial BDS activity by biosurfactants and the determination of which features play more important role in the biocatalysis have not been studied comprehensively. Therefore, it is important to study biosurfactant-producing strains and the combined effects of biosurfactant and the strain on the BDS of DBT. This also settles the problem of low bioavailability of sulfocompounds.

\section{Experimental}

\subsection{Materials}

DBT was purchased from Sigma-Aldrich Co. (USA) and the purity was $98 \%$. HBP was bought from Gracia Chemical 
Technology Co., Ltd. in China and its purity was also 98\%. Other reagents were analytical grade and used without further purification.

\subsection{Culture and bacterial identification}

Desulfurization bacteria were isolated from soil samples collected from petroleum-contaminated grounds at Shengli oilfield and enriched with Luria-Bertani (LB) medium at $50{ }^{\circ} \mathrm{C}$. Then, they were transferred to an oven and kept at $70{ }^{\circ} \mathrm{C}$ for $2 \mathrm{~h}$ to kill the temperature sensitive bacteria. The resulting bacteria were cultured in an incubator at $50{ }^{\circ} \mathrm{C}$. Subsequently, they were washed with $\mathrm{NaCl}$ solution of $0.9 \%$ and inoculated in the basal salt medium (BSM) with an appropriate amount of DBT (0.2 $\mathrm{mmol} \mathrm{L}^{-1}$ ) as a sulfur source. The optimum culture temperature was estimated using the parabola method. The genomic DNA of the strain was extracted with a modified method, i.e., modified sodium dodecyl sulfate (SDS) and cetyl trimethyl ammonium bromide (CTAB) method, and purified by phenol-chloroform solution. Finally, the 16S rDNA PCR was amplified with the universal primer pairs, 27F and 1492R, and sequenced with the Sanger method. The basic local alignment search tool (BLAST) was implemented to build the phylogenetic tree.

\subsection{Extraction, purification and qualitative analysis}

After the strain was incubated in BSM for three days with constant agitation in a baffled flask on a rotary shaker, the cell wall was broken with ultrasonic agitation for 30 minutes, and the cell debris was centrifuged to obtain the liquid supernatant. Subsequently, the $\mathrm{pH}$ value of the supernatant was adjusted to 2.0 with $\mathrm{HCl}$ acid. The flocculent precipitate was then centrifuged to harvest the crude biosurfactant. Component analysis was carried out with ion type identification, thin-layer chromatography (TLC), gas chromatography-mass spectrometry (GC-MS), Fourier transform infrared spectroscopy (FT-IR) and nuclear magnetic resonance spectroscopy (NMR).

\subsection{Property analysis of biosurfactant}

The analysis of emulsifying properties, surface tension and oil repellent capability of the biosurfactant was carried out as follow: the emulsion properties were determined with $E_{24}$, which was calculated using the height percentage of the emulsified layer $(\mathrm{mm})$ on the basis of the total height of the liquid column (mm) ${ }^{7}$ after the supernatant (bacterial lysis solution) and liquid paraffin were mixed for $24 \mathrm{~h}$. The surface tension of the ultrasonic crushing solution and distilled water was measured using the maximum-bubble-pressure method. The oil-repellent capability was determined by the oil-repellent coating film test (stained liquid paraffin).

\subsection{Desulfurization performance of biosurfactant}

The Bacillus sp. Lv13 was equally inoculated into BSM medium with DBT $\left(0.2 \mathrm{mmol} \mathrm{L}^{-1}\right)$. The bacteria were divided into two groups. One group was added with biosurfactant $\left(0.05 \mathrm{~g} \mathrm{~L}^{-1}\right)$ and the other as a control. After 4 days of culture, DBT and its metabolites were extracted from the medium with 1 volume of $n$-hexane. The degradation rate of DBT and HBP was calculated from the respective standard curve. 2-HBP was determined using the Gibbs assay as described in literature ${ }^{\mathbf{1 0}}$ and confirmed by GC-MS. Other derivatives were also characterized by GC-MS analysis (Agilent, HP-5MS column) with the following temperature programme: $100{ }^{\circ} \mathrm{C}$ for $2 \mathrm{~min}, 10{ }^{\circ} \mathrm{C} \mathrm{m^{-1 }}$ to $300{ }^{\circ} \mathrm{C}$, sample injecting temperature was $290{ }^{\circ} \mathrm{C}$. Furthermore, the ingredient in the inorganic sulfide compound could be identified with $\mathrm{BaCl}_{2}$ solution and $\mathrm{HNO}_{3}$ acid.

\section{Results and discussion}

\subsection{Identification of the desulfurization strains}

The 16S rDNA gene has highly conserved sequences that can be used to study the phylogenetic relationship of the prokaryotic organisms. In this comparison, BLAST software was used to search homology sequences in the Gene-Bank database. The Bacillus sp. Lv13 and other 10 sequences were selected to build the phylogenetic tree (Fig. 1) with the MEGA7 software after multiple comparisons (bootstrap $=1000$ ). Based on the results of comparison, the isolated strain exhibited the highest similarity to Bacillus licheniformis and was named as Bacillus sp. Lv13. Therefore, the strain was classified as Bacillus licheniformis. Thermophilic analysis of the strain proved that it was a moderate thermophilic bacterium based on the optimum growth temperature $\left(53{ }^{\circ} \mathrm{C}\right)$ calculated with parabolic method. This finding suggests that it can be used in a variety of desulfurization processes under extreme conditions.

\subsection{Qualitative analysis of the biosurfactant}

The biosurfactant extracted from the Bacillus sp. Lv13 was identified with ion type identification, TLC, GC-MS and FT-IR. For ion type identification, the color was transferred to the lower layer using SDS solution, which indicated that it is a nonionic surfactant. In the coloring process for the TLC, the TLC plate containing the crude products was colored purple after reacting with ninhydrin and dyed yellow with bromothymol blue reagent. According to these results, it must be a kind of lipopeptide. GC-MS and FT-IR were two more accurate methods used to identify the biosurfactant. The results from the GC-MS analysis revealed that more than 10 major congeners

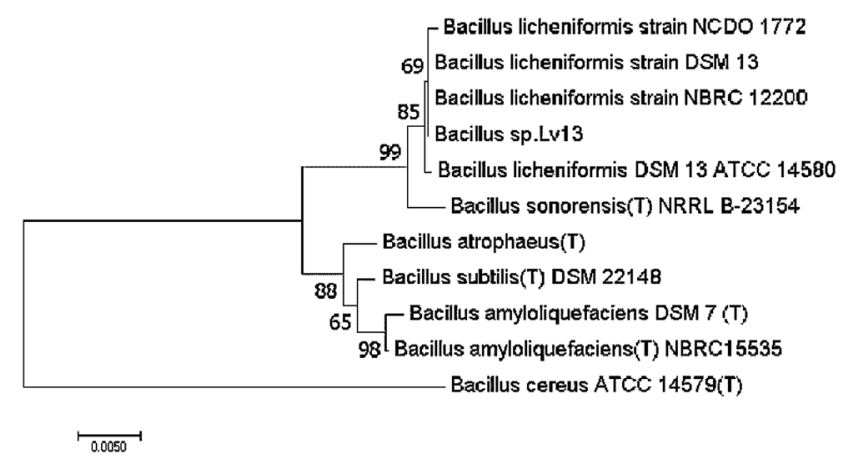

Fig. 1 Genomic identification by $16 \mathrm{~S}$ rRNA sequencing. 
existed in the biosurfactant. The peaks at the retention times of 10.57, 23.75, $27.67 \mathrm{~min}$ are presumed to be associated to 2myristynoyl pantetheine with a molecular weight of $484 \mathrm{Da}$ and the formula of $\mathrm{C}_{25} \mathrm{H}_{44} \mathrm{~N}_{2} \mathrm{O}_{5} \mathrm{~S}$ (Table 1). It is ascribed that the biosurfactants had several homologs, some of which have biosurfactant properties. Lipid-based compounds 2,6,10-trimethyltetradecane (at the retention time of 9.45, $14.64 \mathrm{~min}$ ) and 2,4bis(1,1-dimethylethyl)-phenol (at the retention time of 15.04 min), were possibly the components of solvents or impurities. The FTIR spectrum of the biosurfactant is shown in Fig. 2. The two peaks at 3170 and $3420 \mathrm{~cm}^{-1}$ correspond to $\mathrm{N}-\mathrm{H}$ bonding, which indicates the stretching vibration of the amides. The characteristic peak at $1617 \mathrm{~cm}^{-1}$ accounts for the presence of $\mathrm{N}-\mathrm{H}$ and $\mathrm{C}=\mathrm{O}$ bonding. Peak at $1409 \mathrm{~cm}^{-1}$ is related to cis form of lactam. The GC-MS and FTIR analysis of this biosurfactant further confirmed that it should be classified as a lipopeptide. The results of ${ }^{1} \mathrm{HNMR}$ spectra partially proved the speculation. Because the LP was not dissolved well and interfered with the impurity, we got weak NMR signals. The presence of fatty acids was indicated by characteristic shifts in the region of 0.891.57 ppm. However, the presence of $\mathrm{N}-\mathrm{H}$ could be partially determined by the region of $7.26 \mathrm{ppm}$ in spite of the interference of solvent peak.

\subsection{Properties of the biosurfactant}

The diameter of the repelled oil circle was $0 \mathrm{~cm}$ and $1.8 \mathrm{~cm}$, respectively, for the supernatant and the lysate solution. Which proved that it was a kind of intracellular products. The average emulsification index, $E_{24}$, was $46.93 \%$, which is much higher than the previously reported data for Hamzah whose emulsification index was $25.29 \% .{ }^{11}$ The surface tension of the cell lysate was $69.05 \mathrm{mN} \mathrm{m}^{-1}$, and its critical micelle concentration (CMC) was just $30 \mathrm{mg} \mathrm{L}^{-1}$.

\subsection{Desulfurization performance of the biosurfactant}

According to the GC-MS analysis results (Fig. 3), the yield of HBP was very low at the initial stage without the addition of lipopeptide, and drastically increased after the addition of lipopeptide. This finding suggests that the efficiency of desulfurization was significantly increased by the lipopeptide. The removal rate of DBT was evidently higher than the control in the first day and even reached to $99 \%$ on the fourth day, according to Fig. 4. The yield of HBP was very low without the presence of lipopeptide, but the decrement rate of DBT was

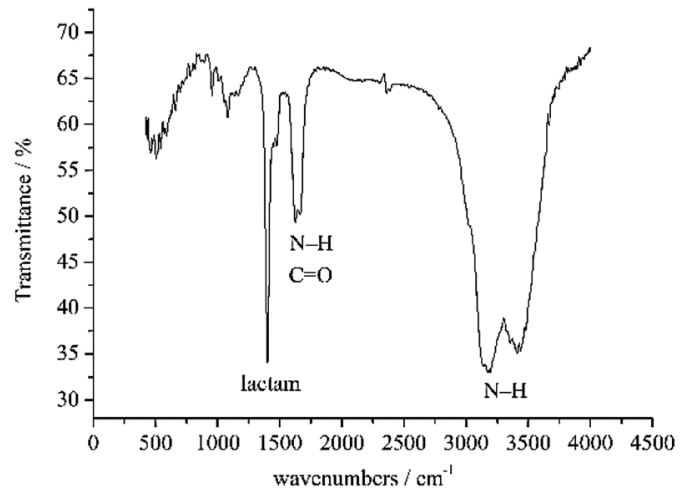

Fig. 2 FTIR spectrum of the biosurfactant.

high. The results are not consistent. The interactions among the DBT, bacteria and biomolecules played a major role and the $4 \mathrm{~S}$ pathway reacted weakly without lipopeptide. After the addition of lipopeptide, the biocatalysis became the major factor in the decrease of DBT (Fig. 5), and the yield of HBP drastically increased. It could be concluded that the addition of lipopeptide increased the capacity of desulfurization, which could be attributed to the following aspects. The emulsification of DBT with the presence of lipopeptide played one important role in the desulfurization of DBT. The longevity of the emulsion layer prolonged the interaction of the bacterial cells and the emulsified DBT, resulting in the improvement of BDS activity. Besides the contact efficiency, the low interface mass transfer was another obstacle for the BDS ${ }^{8}$ because DBT and its metabolites were practically immiscible with water. Lipopeptide not only reduced the oil viscosity, but also increased the mass transfer of DBT. ${ }^{2}$ This facilitated the solubilized DBT to approach the interface of the bacteria and be catalyzed by the enzymes secreted from the bacteria. This can also be explained by their hydrophobic and hydrophilic fragments, which reduced the free energy of the system by replacing the bulk molecules of higher energy at the interface. ${ }^{12}$ Moreover, it could be found that there is a negative correlation between CMC value and the biocatalytic activity in the oil-water biphasic system. The lower the CMC value, the higher the biocatalytic activity. In addition, the lipopeptide had no toxicity to bacterial cell and could be used as an alternative to chemically synthesized surfactants since it showed higher solubilization efficiency towards diesel oil than SDS and Tween $80 .^{13,14}$

Table 1 Major chemical compounds identified by GC-MS analysis

\begin{tabular}{llcll}
\hline RT (min) & Formula & Percentage (\%) & Molecular weight (Da) & Name of compounds (speculated) \\
\hline 9.45 & $\mathrm{C}_{17} \mathrm{H}_{36}$ & 11.3 & 240 & 2,6,10-Trimethyl-tetradecane \\
10.57 & $\mathrm{C}_{25} \mathrm{H}_{44} \mathrm{~N}_{2} \mathrm{O}_{5} \mathrm{~S}$ & 9.42 & 484 & 2-Myristynoyl pantetheine \\
14.64 & $\mathrm{C}_{17} \mathrm{H}_{36}$ & 9.39 & 240 & $2,6,10$-Trimethyl-tetradecane \\
15.04 & $\mathrm{C}_{14} \mathrm{H}_{22} \mathrm{O}$ & 19.3 & 206 & $2,4-$-Bis(1,1-dimethylethyl)-phenol \\
19.41 & $\mathrm{C}_{20} \mathrm{H}_{42} \mathrm{O}_{2}$ & 7.78 & 314 & 2-(Octadecyloxy)-ethanol \\
23.75 & $\mathrm{C}_{25} \mathrm{H}_{44} \mathrm{~N}_{2} \mathrm{O}_{5} \mathrm{~S}$ & 6.04 & 484 & 2-Myristynoyl pantetheine \\
27.67 & $\mathrm{C}_{25} \mathrm{H}_{44} \mathrm{~N}_{2} \mathrm{O}_{5} \mathrm{~S}$ & 5.22 & 484 & 2-Myristynoyl pantetheine
\end{tabular}




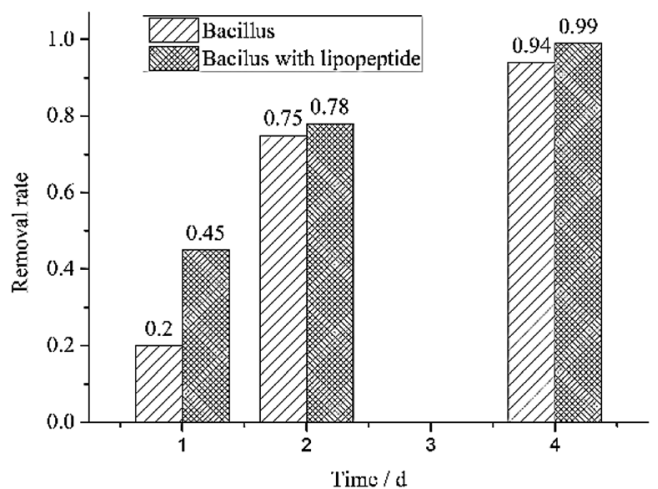

Fig. 3 GC-MS spectra of the BDS process with and without BS.

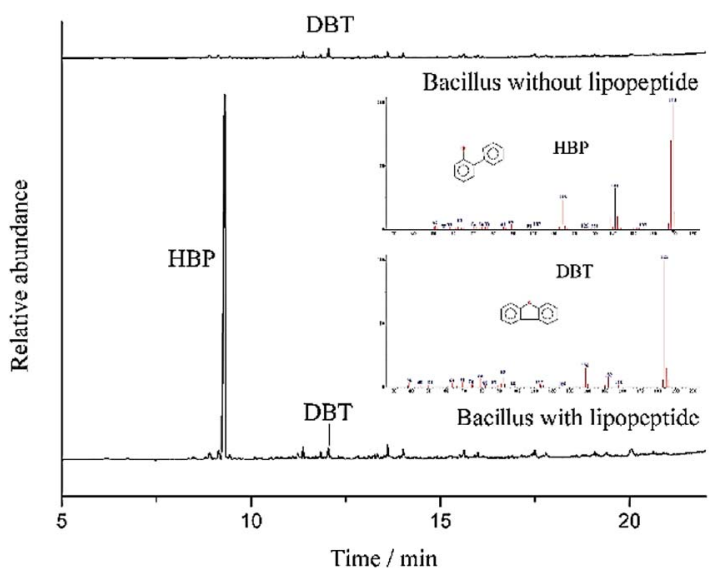

Fig. 4 Removal rate of DBT content with different BDS process at different times.

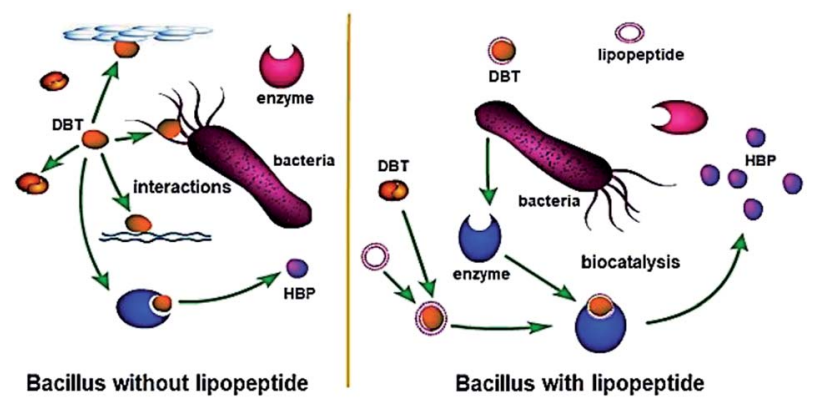

Fig. 5 Patterns of effect of lipopeptide on the improvement of their biocatalysis in BDS.

Other tests also further verified the aforementioned results. Color reactions from the Gibbs assay showed that one of the degradation products of DBT was 2-hydroxybiphenyl. The $\mathrm{BaSO}_{4}$ precipitate test also proved the existence of $\mathrm{SO}_{4}{ }^{2-}$ or $\mathrm{SO}_{3}{ }^{2-}$. These results indicate that the pathway of the biodesulfurization followed the $4 \mathrm{~S}$ pathway. Therefore, the carbon skeleton was not destructed and the calorific value was not lowered in the BDS. It will have great potential uses in environmental treatment in the future.

\section{Conclusions}

Bacillus sp. Lv13, a moderate thermophilic bacterium showed similarity to Bacillus licheniformis, having a high desulfurization capacity. Its biosurfactant must be a kind of lipopeptide, which has better emulsifying property and lower CMC value. These characteristics help to stabilize the emulsion and improve mass transfer. After the addition of lipopeptide into the bacterial reaction system, the yield of HBP significantly increased. Lipopeptide enhanced the capacity of BDS and improved the utilization of the insoluble sulfocompound. The Gibbs assay and $\mathrm{BaSO}_{4}$ precipitate test showed that the desulfurization pattern followed the efficient $4 \mathrm{~S}$ pathway.

\section{Conflicts of interest}

There are no conflicts to declare.

\section{Acknowledgements}

The authors would like to thank Bing Wang and Xiao-hui Dong for their collaboration during the performance analysis of the biosurfactant. This report was supported by the National Natural Science Foundation of China (U1610103) and SDUST Research Fund (2014TDJH105).

\section{References}

1 G. Mohebali, A. Ball, A. Kaytash and B. Rasekh, Stabilization of water/gas oil emulsions by desulfurizing cells of Gordonia alkanivorans RIPI90A, Microbiology, 2007, 153(5), 1573-1581.

$2 \mathrm{~W}$. Li and X. Jiang, Enhancement of bunker oil biodesulfurization by adding surfactant, World J. Microbiol. Biotechnol., 2013, 29(1), 103-108.

3 J. Feng, Y. Zeng, C. Ma, X. Cai, Q. Zhang, M. Tong, B. Yu and $\mathrm{P} . \mathrm{Xu}$, The surfactant Tween 80 enhances biodesulfurization, Appl. Environ. Microbiol., 2006, 72(11), 7390-7393.

4 Y. Al-Wahaibi, S. Joshi, S. Al-Bahry, A. Elshafie, A. Al-Bemani and B. Shibulal, Biosurfactant production by Bacillus subtilis B30 and its application in enhancing oil recovery, Colloids Surf., B, 2014, 114(8), 324-333.

5 H. Liu, H. Wang, X. Chen, N. Liu and S. Bao, Biosurfactantproducing strains in enhancing solubilization and biodegradation of petroleum hydrocarbons in groundwater, Environ. Monit. Assess., 2014, 186(7), 45814589.

6 S. Pansiripat, O. Pornsunthorntawee, R. Rujiravanit, B. Kitiyanan, P. Somboonthanate and S. Chavadej, Biosurfactant production by Pseudomonas aeruginosa SP4 using sequencing batch reactors: Effect of oil-to-glucose ratio, Biochem. Eng. J., 2010, 49(2), 185-191.

7 S. Bandyopadhyay, R. Chowdhury and C. Bhattacharjee, Production of biosurfactants through biodesulfurization of spent engine oil - an experimental study, Green Chem. Lett. Rev., 2014, 7(3), 288-295.

8 H. Kawaguchi, H. Kobayashi and K. Sato, Metabolic engineering of hydrophobic Rhodococcus opacus for 
biodesulfurization in oil-water biphasic reaction mixtures, $J$. Biosci. Bioeng., 2012, 113(3), 360-366.

9 M. Dinamarca, A. Rojas, P. Baeza, G. Espinoza, C. IbacacheQuiroga and J. Ojeda, Optimizing the biodesulfurization of gas oil by adding surfactants to immobilized cell systems, Fuel, 2014, 116(1), 237-241.

10 D. Boniek, D. Figueiredo, V. Pylro and G. Duarte, Characterization of bacterial strains capable of desulphurisation in soil and sediment samples from Antarctica, Extremophiles, 2010, 14(5), 475-481.

11 A. Hamzah, N. Sabturani and S. Radiman, Screening and optimization of biosurfactant production by the hydrocarbon-degrading bacteria, Sains Malays., 2013, 42(5), 615-623.
12 J. Raheb, S. Ghaffari, M. Hajipour, B. Memari and M. Kefayati, The In Vitro Rhamnolipid Biosurfactant Reduced Energy Consuming in Biodesulfurization Activity of Genetically Engineered Strain Pseudomonas aeruginosa ATCC9027, Energy Sources, Part A, 2012, 34(14), 1318-1325.

13 H. Ben Ayed, N. Jemil, H. Maalej, A. Bayoudh, N. Hmidet and M. Nasri, Enhancement of solubilization and biodegradation of diesel oil by biosurfactant from Bacillus amyloliquefaciens An6, Int. Biodeterior. Biodegrad., 2015, 99, 8-14.

14 C. Lai, Y. Huang, Y. Wei and J. Chang, Biosurfactantenhanced removal of total petroleum hydrocarbons from contaminated soil, J. Hazard. Mater., 2009, 167(1-3), 609614. 